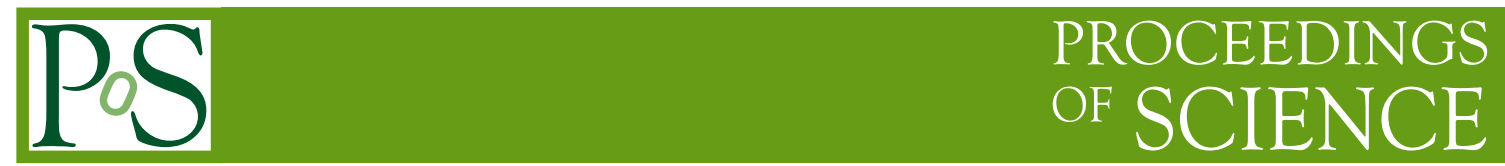

\title{
Search for dark matter in pp collisions at ATLAS
}

\author{
Steven Schramm*, on behalf of the ATLAS Collaboration \\ Universite de Geneve $(\mathrm{CH})$ \\ E-mail: steven.schrammecern.ch
}

Dark Matter comprises approximately $27 \%$ of the universe yet little is known about its properties. Dark matter particles, if produced by the LHC, will leave a distinct signature of significant missing transverse momentum ( $\mathrm{E}_{\mathrm{T}}^{\mathrm{miss}}$ ). Recent results from ATLAS utilising the $\mathrm{E}_{\mathrm{T}}^{\mathrm{miss}}$ signature found in association with a variety of probes will be presented.

38th International Conference on High Energy Physics 3-10 August 2016

Chicago, USA

${ }^{*}$ Speaker. 


\section{Introduction}

The nature of Dark Matter (DM) remains one of the largest open questions in particle physics. Astrophysical experiments observe its gravitational effects on large distance scales, and have measured the universal DM density to be approximately five times that of normal matter, but there is no experimentally confirmed theory of its origin. Leading theories assume DM is a type of Weakly Interacting Massive Particle (WIMP), meaning that it weakly couples to the SM, as motivated by the so-called WIMP miracle where such a particle naturally reproduces the observed DM relic density.

If DM is a WIMP, then the weak coupling between the SM and DM particles should be observable in localized experiments. Numerous searches have been conducted, generally falling into one of three categories: existing DM recoiling off of SM particles (direct detection), SM by-products of galactic DM-DM annihilation (indirect detection), and production of DM in high-energy SM collisions (collider production). The latter category is a key piece of the ATLAS [1] physics program.

ATLAS DM searches revolve around two main strategies. The first approach is the search for events in which DM is pair-produced in association with another object, typically through initial state radiation (ISR). This ISR object is necessary, as the production of DM without other particle(s) is invisible to the detector, while visible object(s) plus DM will appear as a significant imbalance in the transverse momentum $\left(\mathrm{E}_{\mathrm{T}}^{\mathrm{miss}}\right)$ of the event. This class of searches is often referred to as $\mathrm{E}_{\mathrm{T}}^{\text {miss }}+\mathrm{X}$.

The second approach is the search for mediators which could connect the SM to the DM. This strategy is useful as any SM process that can form the dark mediator can also decay back to the input SM particles, so long as the mediator is sufficiently massive. The most common example is the search for dijet resonances, where it is assumed that the mediator couples to light quarks or gluons, and an observed resonance could be interpreted as the production of a new dark mediator.

In interpreting results from searches for dark matter or dark mediators, ATLAS primarily relies on simplified models connecting the two SM inputs to the two DM outputs via a single vector, axialvector, or scalar mediator. Using such a simplified model means that dark matter and dark mediator searches are directly comparable. All results presented will make use of such simplified models.

\section{Dark matter production searches}

ATLAS searches for the direct production of DM focus on the $\mathrm{E}_{\mathrm{T}}^{\mathrm{miss}}+\mathrm{X}$ topology, where the $\mathrm{X}$ represents object(s) that interact with the ATLAS detector. These objects are typically from ISR sources, although in some cases they may come from a mixing with the mediator. No statistically significant excess beyond the SM is observed in any of the topologies to be discussed, and thus they all proceed to set limits on the production of DM.

The final state with the highest SM cross-section is the $\mathrm{E}_{\mathrm{T}}^{\mathrm{miss}}+\mathrm{jet}(\mathrm{s})$ analysis, where the large ISR jet production rate is exploited to obtain the strongest vector and axial-vector mediator limits. The primary background is the irreducible $\mathrm{Z}(\rightarrow v v)+$ jets process, followed by $\mathrm{W}(\rightarrow \ell v)+$ jets. Corresponding limits on axial-vector mediators for the 2015 dataset are shown in Figure 1.

The $\mathrm{E}_{\mathrm{T}}^{\text {miss }}$ +photon analysis has a smaller SM cross-section, as ISR photons are less common at the LHC, but is a much cleaner final state. The primary background is the irreducible $\mathrm{Z}(\rightarrow v v)+\gamma$ process, followed by $\mathrm{W}(\rightarrow \ell v)+\gamma$. Corresponding limits on axial-vector mediators for the 2015 dataset are shown in Figure 1. 

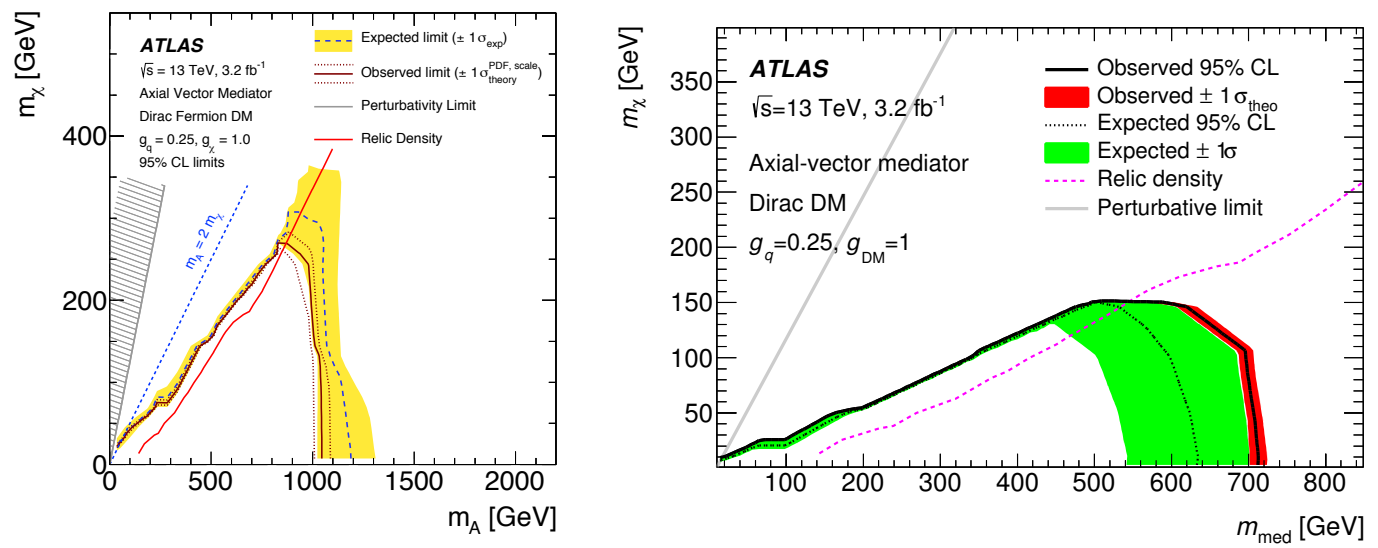

Figure 1: Limits on an axial-vector mediator simplified model for the production of DM, with a variable mediator mass $m_{A}\left(m_{\text {med }}\right)$, a variable DM mass $m_{\chi}$, a quark coupling $g_{q}$ fixed to 0.25 , and a DM coupling $g_{\chi}\left(g_{\mathrm{DM}}\right)$ fixed to 1 , from the (left) $\mathrm{E}_{\mathrm{T}}^{\mathrm{miss}}+\mathrm{jet}[2]$ and (right) $\mathrm{E}_{\mathrm{T}}^{\mathrm{miss}}+$ photon [3] analyses.

The $\mathrm{E}_{\mathrm{T}}^{\mathrm{miss}}+\mathrm{Z}(\rightarrow \ell \ell)$ final state is an even cleaner analysis, where the $\mathrm{Z}$ comes from ISR. However, this comes with a further reduction in the amount of statistics, meaning that it is not currently as sensitive as the two aforementioned analyses. The primary background is the irreducible $\mathrm{Z}(\rightarrow \ell \ell) \mathrm{Z}(\rightarrow v v)$ process, followed by other diboson production processes. Corresponding limits on vector mediators for the 2015 and early 2016 datasets are shown in Figure 2.

While not as clean as the $\mathrm{E}_{\mathrm{T}}^{\text {miss }}+\mathrm{Z}(\rightarrow \ell \ell)$ final state, the boosted $\mathrm{E}_{\mathrm{T}}^{\text {miss }}+\mathrm{W} / \mathrm{Z}(\rightarrow q q)$ analysis has more statistics. The $\mathrm{W}(\mathrm{Z})$ decays hadronically three times (ten times) more often than to electrons or muons, providing a boost in statistical power. However, the background also grows considerably, and the identification of hadronic decays is not as clean as leptonic decays. The irreducible $\mathrm{Z} \rightarrow v v+$ jets process remains dominant, where the jet fakes a W/Z hadronic decay. Corresponding limits on vector mediators for the 2015 dataset are shown in Figure 2. Different sized datasets are used for this analysis compared to the $\mathrm{E}_{\mathrm{T}}^{\mathrm{miss}}+\mathrm{Z}(\rightarrow \ell \ell)$ analysis, so sensitivity cannot be directly compared.
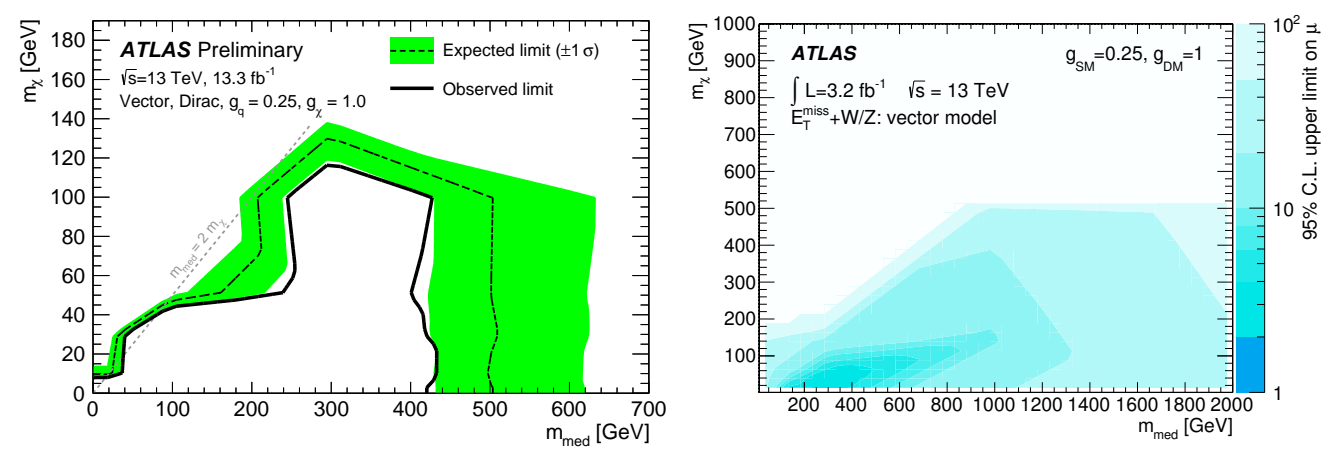

Figure 2: Limits on a vector mediator simplified model for the production of DM, with a variable mediator mass $m_{\text {med }}$, a variable DM mass $m_{\chi}$, a quark coupling $g_{q}\left(g_{\mathrm{SM}}\right)$ fixed to 0.25 , and a DM coupling $g_{\chi}\left(g_{\mathrm{DM}}\right)$ fixed to 1 , from the $($ left $) \mathrm{E}_{\mathrm{T}}^{\mathrm{miss}}+\mathrm{Z}(\rightarrow \ell \ell)[4]$ and (right) $\mathrm{E}_{\mathrm{T}}^{\mathrm{miss}}+\mathrm{W} / \mathrm{Z}(\rightarrow q q)$ [5] analyses. 
An interesting alternative is to consider the $\mathrm{E}_{\mathrm{T}}^{\text {miss }}+\mathrm{H}$ topology, where the Higgs boson mixes with the mediator and thus does not come from ISR. The first final state considered is $\mathrm{H} \rightarrow b b$, which has the largest cross-section. This final state has two analyses, one for $\mathrm{E}_{\mathrm{T}}^{\mathrm{miss}}<500 \mathrm{GeV}$ or the resolved regime, and one for $\mathrm{E}_{\mathrm{T}}^{\mathrm{miss}}>500 \mathrm{GeV}$ or the boosted regime. In both cases, the dominant backgrounds are $\mathrm{W} / \mathrm{Z}+$ jets and especially $t \bar{t}$. Corresponding limits on vector mediators for the 2015 dataset are shown in Figure 3.

The second $\mathrm{E}_{\mathrm{T}}^{\mathrm{miss}}+\mathrm{H}$ final state considered is $\mathrm{H} \rightarrow \gamma \gamma$, which is much cleaner but also has much lower statistics. This analysis uses a data-driven background fit, and has a primary background from the SM $\gamma \gamma$ and $\gamma+$ jet processes. Corresponding limits on vector mediators for the 2015 and early 2016 datasets are shown in Figure 3. Different sized datasets are used for this analysis compared to the $\mathrm{E}_{\mathrm{T}}^{\mathrm{miss}}+\mathrm{H}(\rightarrow b b)$ analysis, so sensitivity cannot be directly compared.
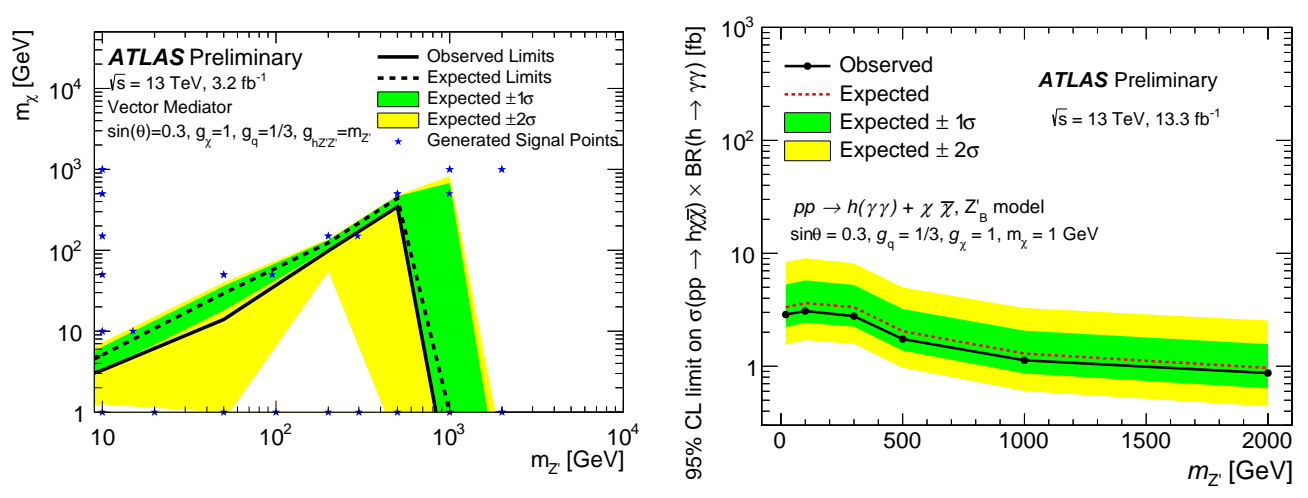

Figure 3: Limits on a vector mediator simplified model for the production of DM, with a variable mediator mass $m_{Z^{\prime}}$, a DM mass $m_{\chi}$, a quark coupling $g_{q}$ fixed to $1 / 3$, a DM coupling $g_{\chi}$ fixed to 1 , and a mixing parameter $\sin \theta$ fixed to 0.3 , from the (left) $\mathrm{E}_{\mathrm{T}}^{\text {miss }}+\mathrm{H}(\rightarrow b b)$ [6] and (right) $\mathrm{E}_{\mathrm{T}}^{\text {miss }}+\mathrm{H}(\rightarrow \gamma \gamma)$ [7] analyses. The $\mathrm{H} \rightarrow b b$ channel uses a variable DM mass, while the $\mathrm{H} \rightarrow \gamma \gamma$ channel fixes the DM mass to $1 \mathrm{GeV}$.

The $\mathrm{E}_{\mathrm{T}}^{\mathrm{miss}}+\mathrm{bb}$ analysis is particularly sensitive to scalar mediators due to the expected dependence of the scalar coupling on the quark mass, which is another interesting possibility to consider. At the LHC, the di-b-jet production diagram dominates the single-b-jet diagram, which is why the analysis searches for di-b-jet final states. Primary backgrounds include top and W/Z+jets, where the former is suppressed by requiring exactly two jets in the event. Corresponding limits on scalar mediators for the 2015 and early 2016 datasets are shown in Figure 4.

\section{Dark mediator production searches}

ATLAS searches for dark mediator production are primarily realized in the form of dijet resonance searches. This constrains the search to mediators which couple to light quarks or gluons, and in the absence of any such resonance, limits are set on the coupling to new physics. This can be interpreted as a limit on the production of DM mediators for a given choice of couplings.

While $\mathrm{E}_{\mathrm{T}}^{\mathrm{miss}}+\mathrm{X}$ searches are sensitive to a wide range of mediator masses, dijet searches have a lower boundary defined by the trigger. The standard dijet search using the 2015 and early 2016 datasets is limited to mediator masses above approximately $1.5 \mathrm{TeV}$, as shown in Figure 5. 
In order to probe lower mediator masses, alternative triggering approaches are needed. ATLAS has studied two main approaches, the first of which is referred to as dijet+ISR searches. This analysis requires that the trigger fire off of a third object, assumed to be an ISR jet or photon, and which balances the dijet system. The ISR jet option allows for an enhanced sensitivity over a small kinematic range, while the ISR photon final state provides a bit less sensitivity but covers a much larger kinematic range. With these two approaches, the limits can be set on the production of dark mediators down to approximately $200 \mathrm{GeV}$, as seen in Figure 5.

The other strategy employed by ATLAS is referred to as the dijet Trigger Level Analysis (TLA). In this scenario, a different approach is used. The trigger system is limited by the amount of bandwidth that has to be written out, where bandwidth is the size of the event multiplied by the rate (number of events per second). In order to write out events at a rate approximately 100 times higher than for normal jet triggers, only a very small part of the event is written out, leading to a much reduced event size. This allows for full access to mediator masses down to approximately $450 \mathrm{GeV}$. While this does not have the same kinematic reach as the dijet+ISR method described above, the amount of statistics is dramatically increased, leading to much higher sensitivity and thus stronger limits where the TLA is available, as shown in Figure 5.

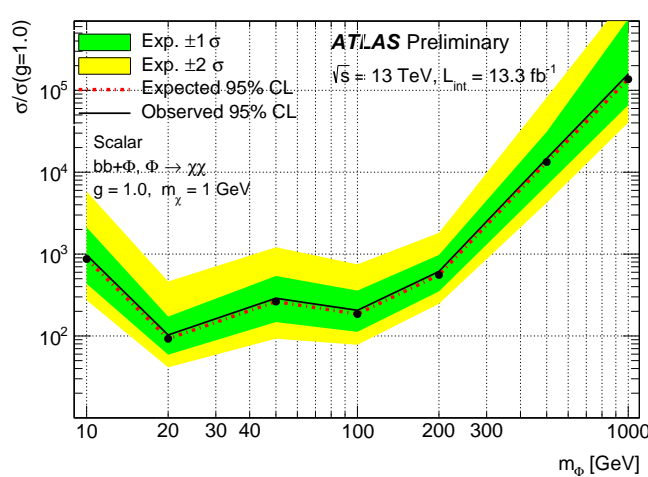

Figure 4: Limits on a scalar mediator simplified model for the production of DM, with a variable mediator mass $m_{\Phi}$, a DM mass $m_{\chi}$ fixed to $1 \mathrm{GeV}$, and a coupling $g$ fixed to 1 , from the $\mathrm{E}_{\mathrm{T}}^{\mathrm{miss}}+\mathrm{bb}$ analysis [8].

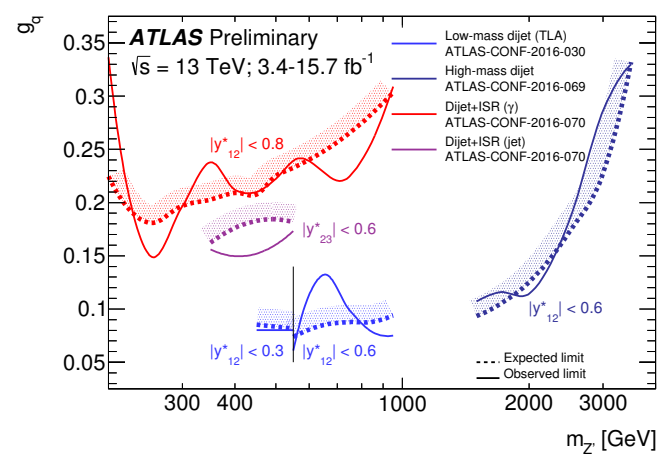

Figure 5: A summary of ATLAS limits on the production of lepto-phobic axial vector mediators that can be re-interpreted in the context of simplified models for the production of DM, with a variable mediator mass $m_{Z^{\prime}}$ and a variable quark coupling $g_{q}[9]$.

The three dijet analysis strategies are re-interpreted in terms of dark mediators under two different coupling assumptions in Figure 6. Under one set of coupling assumptions, the dijet searches cover the vast majority of the parameter space considered, leaving only small contributions from the $\mathrm{E}_{\mathrm{T}}^{\mathrm{miss}}+\mathrm{X}$ analyses. However, a small change in the coupling assumptions completely changes the picture, with the dijet searches ruling out a much smaller part of parameter space and the $\mathrm{E}_{\mathrm{T}}^{\mathrm{miss}}+\mathrm{X}$ analyses covering a complementary region. This demonstrates how both dark matter searches and dark mediator searches are important to covering the possible DM parameter space.

\section{Summary}

The search for Dark Matter (DM) is a key piece of the ATLAS physics program, and is realized through two complementary approaches: dark matter production and dark mediator produc- 

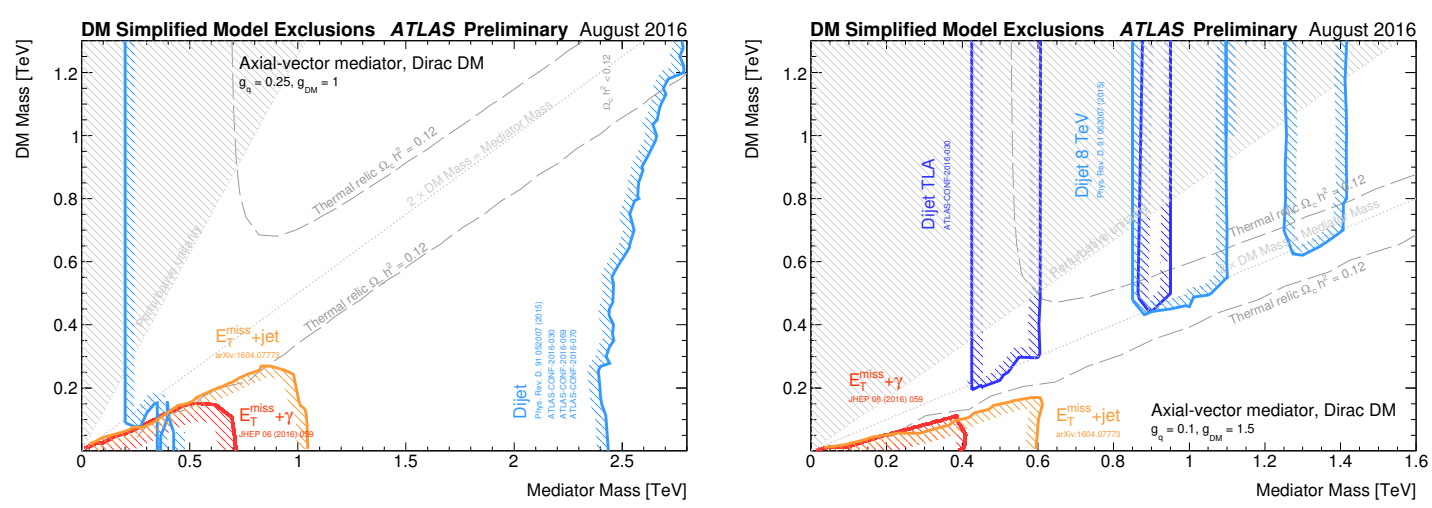

Figure 6: A summary of ATLAS limits on the lepto-phobic axial vector mediators coupling to DM, with variable mediator and DM masses, from both the leading $\mathrm{E}_{\mathrm{T}}^{\mathrm{miss}}+\mathrm{X}$ analyses and dark mediator searches. Coupling values are fixed to (left) 0.25 for quarks and 1 for DM or (right) 0.1 for quarks and 1.5 for DM [9].

tion. The former is primarily studied by $\mathrm{E}_{\mathrm{T}}^{\mathrm{miss}}+\mathrm{X}$ topologies where the $\mathrm{DM}$ is balanced by visible particle(s) X, while the latter is probed by dijet resonance searches. No evidence of physics beyond the Standard Model is observed in either case, and limits are set on vector, axial-vector, and scalar simplified models of DM production.

\section{References}

[1] ATLAS collaboration, ATLAS Experiment at the CERN Large Hadron Collider, JINST 3 (2008) 003.

[2] ATLAS collaboration, Search for new phenomena in final states with an energetic jet and large missing transverse momentum in pp collisions at $\sqrt{s}=13 \mathrm{TeV}$ using the ATLAS detector, Phys. Rev. D94 (2016) 032005, [1604.07773].

[3] ATLAS collaboration, Search for new phenomena in events with a photon and missing transverse momentum in pp collisions at $\sqrt{s}=13 \mathrm{TeV}$ with the ATLAS detector, JHEP 06 (2016) 059, [1604.01306].

[4] ATLAS collaboration, Search for new phenomena in the $Z(\rightarrow \ell \ell)+E_{\mathrm{T}}^{\text {miss }}$ final state at $\sqrt{s}=13 \mathrm{TeV}$ with the ATLAS detector, ATLAS-CONF-2016-056 (2016).

[5] ATLAS collaboration, Search for dark matter produced in association with a hadronically decaying vector boson in pp collisions at $\sqrt{s}=13 \mathrm{TeV}$ with the ATLAS detector, arXiv:1608.02372 [hep-ex].

[6] ATLAS collaboration, Search for Dark Matter in association with a Higgs boson decaying to b-quarks in pp collisions at $\sqrt{s}=13 \mathrm{TeV}$ with the ATLAS detector, ATLAS-CONF-2016-019 (2016).

[7] ATLAS collaboration, Search for new phenomena in events with missing transverse momentum and a Higgs boson decaying to two photons in pp collisions at $\sqrt{s}=13 \mathrm{TeV}$ with the ATLAS detector, ATLAS-CONF-2016-087 (2016).

[8] ATLAS collaboration, Search for Dark Matter production associated with bottom quarks with $13.3 \mathrm{fb}^{-1}$ of pp collisions at $\sqrt{s}=13 \mathrm{TeV}$ with the ATLAS detector at the LHC, ATLAS-CONF-2016-086 (2016).

[9] ATLAS Collaboration, "Summary plots from the ATLAS Exotic physics group." https://atlas.web.cern.ch/Atlas/GROUPS/PHYSICS/CombinedSummaryPlots/EXOTICS/. 